\title{
Epidemiologic update on the dengue situation in the Western Pacific Region, 2011
}

Yuzo Arima, ${ }^{a}$ Zoe Rebecca Edelstein, ${ }^{a}$ Hwi Kwang Han ${ }^{a}$ and Tamano Matsui a on behalf of the Emerging Disease Surveillance and Response Team, Division of Health Security and Emergencies, World Health Organization Regional Office for the Western Pacific

Correspondence to Yuzo Arima (e-mail: arimay@wpro.who.int).

Dengue is an emerging vectorborne infectious disease that is a major public health concern in Asia and the Pacific. Official dengue surveillance data for 2011 provided by ministries of health were summarized as part of routine activities of the World Health Organization Regional Office for the Western Pacific. Based on officially reported surveillance data, dengue continued to show sustained activity in the Western Pacific Region. In 2011, Member States reported a total of 244855 cases of which 839 died. More than 1000 cases were reported each from Cambodia, the Federated States of Micronesia, the Lao People's Democratic Republic, Malaysia, the Philippines, the Marshall Islands, Singapore and Viet Nam. Cambodia, the Federated States of Micronesia and the Marshall Islands reported more cases relative to 2010. There continues to be great variability among the dengue-endemic countries and areas in the Region in the number of cases and serotype distribution. The continued high notification rate and complex dengue epidemiology in the Region highlight the need for information-sharing on a routine and timely basis.

D engue, an emerging arboviral infection, continues to cause a substantial public health burden in Asia and the Pacific. Thanks to continuous, and increasingly more reliable and systematic, dengue surveillance systems in many dengue-endemic countries in the Western Pacific Region, dengue has shown not only its high burden but also its complex epidemiology of seasonality, multiyear oscillations and varying age, gender and serotype distributions over time. ${ }^{1-4}$ Substantial economic costs have also been associated with the disease at the household and overall economy levels, ${ }^{5,6}$ highlighting the continued need to respond to this threat. The reported number of dengue cases have increased over the past decade; since 2007, more than 200000 cases have been consistently reported in the Region annually.

In 2010, there were 353907 dengue cases and 1073 dengue deaths reported from 24 of 37 countries and areas in the Western Pacific Region. ${ }^{7}$ Countries that reported more than 1000 cases were Australia, Cambodia, the Lao People's Democratic Republic, Malaysia, the Philippines, Singapore and Viet Nam. Cambodia, the Lao People's Democratic Republic, Malaysia, the Philippines, Singapore and Viet Nam contributed 1070 of the reported 1073 deaths.
Continuous and systematic dengue surveillance is variable and limited among the Pacific island countries and areas; however, dengue cases continued to be reported. In 2010, there were fewer dengue notifications relative to 2009; relatively high notification rates were reported from American Samoa (77/100 000 population), French Polynesia (92/100 000 population) and Vanuatu (78/100 000 population). Dengue surveillance is not conducted in Papua New Guinea but circulation of the virus there is well known given the importation of cases into Australia. ${ }^{8}$

Based on officially reported national surveillance data, the World Health Organization (WHO) Regional Office for the Western Pacific communicates the latest annual regional dengue situation.

\section{METHODS}

This report provides a descriptive summary of dengue cases reported in 2011 from surveillance systems from countries and areas in the Western Pacific Region.

Further data are provided from Australia, Cambodia, the Lao People's Democratic Republic, Malaysia, the Philippines, Singapore and Viet Nam;

\footnotetext{
Emerging Disease Surveillance and Response, Division of Health Security and Emergencies, World Health Organization Regional Office for the Western Pacific, Manila, Philippines. 
Table 1. Dengue case definitions, laboratory sampling and testing methods used for surveillance in Cambodia, the Lao People's Democratic Republic, Malaysia, the Philippines, Singapore, Viet Nam and Australia, 2011

\begin{tabular}{|c|c|c|c|}
\hline \multirow[b]{2}{*}{ Country } & \multicolumn{2}{|l|}{ Case definition } & \multirow[b]{2}{*}{ Laboratory sampling and testing method } \\
\hline & Clinical criteria* & $\begin{array}{l}\text { Laboratory } \\
\text { confirmation }\end{array}$ & \\
\hline Australia & $\begin{array}{l}\text { Fever, headache, arthralgia, myalgia, } \\
\text { rash, nausea and vomiting }\end{array}$ & Required & $\begin{array}{l}\text { All clinically diagnosed cases are laboratory tested } \\
\text { and only those confirmed by the following method are } \\
\text { registered: isolation/detection of dengue virus OR IgG } \\
\text { seroconversion or significant increase in antibody level } \\
\text { or } \geq 4 \text {-fold rise in titre to dengue virus OR detection } \\
\text { of dengue virus-specific IgM in cerebrospinal fluid OR } \\
\text { detection of dengue virus-specific IgM in serum }\end{array}$ \\
\hline Cambodia & 2009 dengue case classification ${ }^{\dagger}$ & No & $\begin{array}{l}5 \text { sentinel sites send maximum of } 5 \text { samples per week } \\
\text { for testing, focusing primarily on children. Confirmation is } \\
\text { based on enzyme-linked immunosorbent assay (ELISA), } \\
\text { polymerase chain reaction (PCR) and/or virus isolation. }\end{array}$ \\
\hline $\begin{array}{l}\text { Lao People's } \\
\text { Democratic } \\
\text { Republic }\end{array}$ & 2009 dengue case classification ${ }^{\dagger}$ & No & $\begin{array}{l}\text { A proportion of dengue cases, such as ad hoc outbreak } \\
\text { specimens, are tested by ELISA. }\end{array}$ \\
\hline Malaysia & $\begin{array}{l}\text { Acute onset of high fever } \geq 2-5 \text { days } \\
\text { with } \geq 2 \text { of following: headache, retro- } \\
\text { orbital pain, myalgia, arthralgia, rash } \\
\text { and mild haemorrhagic manifestation }\end{array}$ & No & $\begin{array}{l}\text { A proportion of dengue cases are tested: detection of } \\
\text { dengue lgM/lgG from serum; } \geq 4 \text {-fold rise in IgG/lgM } \\
\text { antibody titres to dengue virus antigen(s) in paired serum } \\
\text { samples; isolation of dengue virus; detection of viral } \\
\text { sequences by PCR. }\end{array}$ \\
\hline Philippines & $\begin{array}{l}\text { Acute onset of fever } 2-7 \text { days with } \\
\geq 2 \text { of following: headache, retro- } \\
\text { orbital pain, myalgia, arthralgia, } \\
\text { rash, haemorrhagic manifestations, } \\
\text { leucopenia }\end{array}$ & No & $\begin{array}{l}\text { A proportion of dengue cases, such as ad hoc outbreak } \\
\text { and cluster specimens, are tested by serology (IgM), and } \\
\text { a limited number by PCR. }\end{array}$ \\
\hline Singapore & $\begin{array}{l}\text { Acute onset of fever } 2-7 \text { days with } \\
\geq 2 \text { of following: headache, backache, } \\
\text { myalgia, rash, retro-orbital pain, } \\
\text { bleeding, leucopenia }\end{array}$ & Required & $\begin{array}{l}\text { All clinically diagnosed cases are laboratory tested and } \\
\text { only those positive by serology (IgM) or PCR/NS-1 are } \\
\text { registered. }\end{array}$ \\
\hline Viet Nam & 2009 dengue case classification ${ }^{\dagger}$ & No & $\begin{array}{l}\text { A proportion of dengue cases are tested through } \\
\text { serology and a limited number by virus isolation. }\end{array}$ \\
\hline
\end{tabular}

Only the minimum criteria required for fulfilling a clinical dengue case definition are included here; additional signs and symptoms required for more severe forms (e.g. dengue haemorrhagic fever, dengue shock syndrome) are not listed here

A probable dengue case is defined as any case with fever and two or more of the following: nausea, vomiting; rash; aches and pains; positive tourniquet test; leucopenia; any warning sign. A case with warning signs are defined as a clinically diagnosed case with any of the following warning signs: abdominal pain or tenderness; persistent vomiting; clinical fluid accumulation; mucosal bleeding; lethargy, restlessness; liver enlargement $>2 \mathrm{~cm}$; increase in hematocrit concurrent with rapid decrease in platelet count. Severe dengue is defined as severe plasma leakage leading to any of the following: shock; fluid accumulation with respiratory distress OR severe bleeding as evaluated by clinician OR severe organ involvement of liver (aspartate amino transferase or alanine amino transferase $\geq 1000$ ), central nervous system (impaired consciousness) or heart and other organs.

their dengue surveillance systems, case definitions, laboratory sampling methodologies and serotype data are described (Table 1). A brief summary of the dengue outbreaks in the Federated States of Micronesia and the Marshall Islands is included.

\section{RESULTS}

\section{Dengue Situation in the Western Pacific Region}

In 2011, Western Pacific Member States reported a total of 244880 cases of which 839 died (Figure 1). In the Asia subregion, both the notification rate and the absolute number of reported dengue cases were highest in the Philippines (Table 2). Although reporting was not complete for the Pacific subregion, more than 1000 cases were reported from the Marshall Islands and the Federated States of Micronesia in late 2011 (Table 2). In New Zealand, 42 cases were reported in 2011 with 41 of these travelling overseas during the incubation period of the disease; seven of these cases (17\%) were reported from Thailand, Indonesia and Malaysia.

Among dengue-endemic countries with routine dengue surveillance and reporting systems (and 
Figure 1. Number of reported dengue cases and case fatality rates (CFR) in the Western Pacific Region, 1991 to 2011

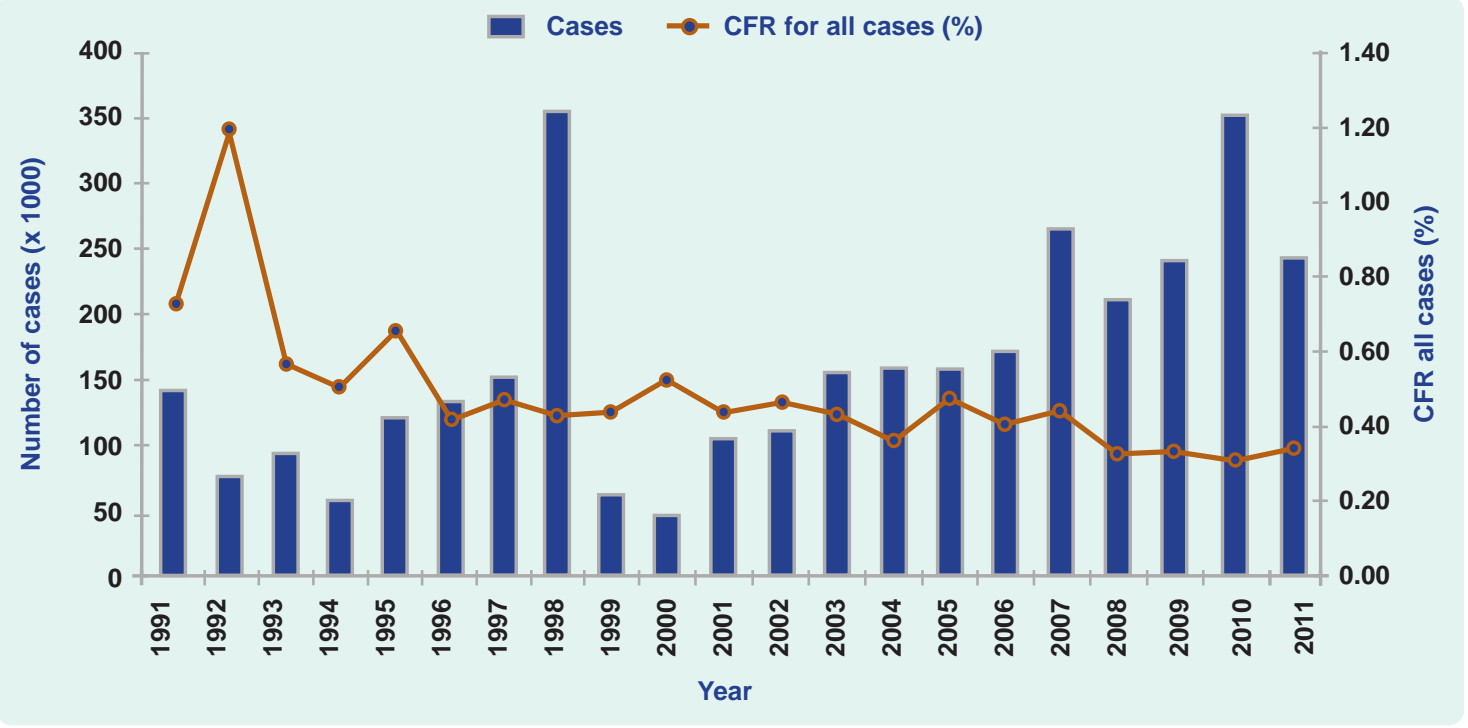

Source: World Health Organization Western Pacific Regional Office based on data provided by the Member States.

Note: Dengue surveillance and reporting systems vary by country.

Table 2. Cases of dengue, including imported cases, and dengue-attributed deaths in the Western Pacific Region, for $2011^{*}$

\begin{tabular}{|c|c|c|c|c|c|}
\hline Countries/territories $^{\dagger}$ & Cases & $\begin{array}{l}\text { Notification } \\
\text { (per } 100000)\end{array}$ & Deaths & $\begin{array}{l}\text { Case fatality } \\
\text { rate (\%) }\end{array}$ & $\begin{array}{l}\text { Population } \\
\text { (x 1000) }\end{array}$ \\
\hline \multicolumn{6}{|l|}{ Asia subregion } \\
\hline Brunei Darussalam & 25 & 6.16 & 0 & 0 & 406 \\
\hline Cambodia & 15980 & 119.29 & 73 & 0.46 & 13396 \\
\hline China & 124 & 0.01 & 0 & 0 & 1370537 \\
\hline Hong Kong (China) & 30 & 0.42 & 0 & 0 & 7068 \\
\hline Japan & 104 & 0.08 & 0 & 0 & 128056 \\
\hline Republic of Korea & 72 & 0.15 & 0 & 0 & 48875 \\
\hline Lao People's Democratic Republic & 3905 & 63.72 & 7 & 0.18 & 6128 \\
\hline Macao (China) & 3 & 0.54 & 0 & 0 & 552 \\
\hline Malaysia & 19884 & 70.38 & 36 & 0.18 & 28251 \\
\hline Mongolia & 0 & 0.00 & 0 & 0 & 2780 \\
\hline Philippines & 125975 & 134.00 & 654 & 0.52 & 94013 \\
\hline Singapore & 5330 & 102.82 & 6 & 0.11 & 5184 \\
\hline Viet Nam & 69680 & 81.00 & 61 & 0.09 & 86025 \\
\hline Total for subregion & 241112 & 13.46 & 837 & 0.35 & 1791271 \\
\hline \multicolumn{6}{|l|}{ Pacific subregion } \\
\hline Australia & 820 & 3.67 & 0 & 0 & 22342 \\
\hline Cook Islands & 0 & 0.00 & 0 & 0 & 23 \\
\hline Fiji & 245 & 28.69 & 0 & 0 & 854 \\
\hline French Polynesia & 12 & 4.46 & 0 & 0 & 269 \\
\hline Marshall Islands & 1257 & 2327.78 & 0 & 0 & 54 \\
\hline Federated States of Micronesia & 1024 & 994.17 & 2 & 0.20 & 103 \\
\hline New Caledonia & 1 & 0.41 & 0 & 0 & 246 \\
\hline New Zealand & 42 & 1.01 & 0 & 0 & 4143 \\
\hline Palau & 334 & 1590.48 & 0 & 0 & 21 \\
\hline Vanuatu & 33 & 14.10 & 0 & 0 & 234 \\
\hline Total for subregion & 3768 & 13.32 & 2 & 0.05 & 28289 \\
\hline TOTAL & 244880 & 13.46 & 839 & 0.34 & 1819560 \\
\hline
\end{tabular}

Source: World Health Organization Western Pacific Regional Office based on data provided by the Member States.

* Dengue surveillance and reporting systems vary by country.

The following countries and areas did not report dengue data: American Samoa, Guam, Kiribati, Nauru, Niue, the Commonwealth of the Northern Mariana Islands, Papua New Guinea, Pitcairn Islands, Samoa, Solomon Islands, Tokelau, Tonga, Tuvalu, and Wallis and Futuna. 
Table 3. Reported number of dengue cases, deaths, and case fatality rates (CFRs) from Cambodia, the Lao People's Democratic Republic, Malaysia, Philippines, Singapore, Viet Nam and Australia, 2007 to $2011^{*}$

\begin{tabular}{|c|c|c|c|c|c|c|c|c|c|c|c|c|c|c|c|}
\hline \multirow[b]{2}{*}{ Country } & \multicolumn{3}{|c|}{2007} & \multicolumn{3}{|c|}{2008} & \multicolumn{3}{|c|}{2009} & \multicolumn{3}{|c|}{2010} & \multicolumn{3}{|c|}{2011} \\
\hline & Cases & Deaths & $\begin{array}{l}\text { CFR } \\
(\%)\end{array}$ & Cases & Deaths & $\begin{array}{l}\text { CFR } \\
(\%)\end{array}$ & Cases & Deaths & $\begin{array}{l}\text { CFR } \\
(\%)\end{array}$ & Cases & Deaths & $\begin{array}{l}\text { CFR } \\
(\%)\end{array}$ & Cases & Deaths & $\begin{array}{l}\text { CFR } \\
\text { (\%) }\end{array}$ \\
\hline Australia & 316 & 0 & 0 & 56 & 0 & 0 & 1401 & 0 & 0 & 1171 & 0 & 0 & 820 & 0 & 0 \\
\hline $\begin{array}{l}\text { Cambodia } \\
\text { Lao }\end{array}$ & 39851 & 407 & 1.02 & 9542 & 65 & 0.68 & 11699 & 38 & 0.32 & 12500 & 38 & 0.30 & 15980 & 73 & 0.46 \\
\hline $\begin{array}{l}\text { People's } \\
\text { Democratic } \\
\text { Republic }\end{array}$ & 4943 & 4 & 0.08 & 4149 & 21 & 0.51 & 7214 & 12 & 0.17 & 22929 & 46 & 0.20 & 3905 & 7 & 0.18 \\
\hline Malaysia & 48846 & 98 & 0.20 & 49335 & 112 & 0.23 & 41486 & 88 & 0.21 & 46171 & 134 & 0.29 & 19884 & 36 & 0.18 \\
\hline Philippines & 55639 & 533 & 0.96 & 39620 & 373 & 0.94 & 57819 & 548 & 0.95 & 135355 & 793 & 0.59 & 125975 & 654 & 0.52 \\
\hline Singapore & 8826 & 24 & 0.27 & 7031 & 10 & 0.14 & 4497 & 8 & 0.18 & 5363 & 6 & 0.11 & 5330 & 6 & 0.11 \\
\hline Viet Nam & 104393 & 88 & 0.08 & 96451 & 97 & 0.10 & 105370 & 87 & 0.08 & 128831 & 55 & 0.04 & 69680 & 61 & 0.09 \\
\hline Total & 262814 & 1154 & 0.44 & 206692 & 678 & 0.33 & 229486 & 781 & 0.34 & 352321 & 1070 & 0.30 & 241574 & 837 & 0.35 \\
\hline
\end{tabular}

* Source: World Health Organization Western Pacific Regional Office based on data provided by the Member States.

* Dengue surveillance and reporting systems vary by country.

Australia where the dengue vectors Aedes aegypti and Aedes albopictus are present and cases occur in North Queensland and the Torres Strait Islands), there has been sustained occurrence of dengue cases over the past five years (Table 3). Except for Cambodia, the number of reported cases in 2011 was less than that of 2010 for these countries.

\section{Asia subregion}

\section{Cambodia}

Under the National Dengue Control Programme, all government health facilities report all clinically suspected dengue cases, and other sites also report passively. In 2011, Cambodia incorporated the 2009 dengue case classification system (Table 1 ) and reported 15980 cases ( 73 fatal) with a peak in week 29 ( $n=854$ cases) in July (Figure 2). Males made up a higher proportion of the reported adolescent and adult cases relative to females. Among 422 laboratorytested cases from five sentinel hospitals, 245 (58\%) were confirmed by enzyme linked immunosorbent assay (ELISA), polymerase chain reaction (PCR) or virus isolation. While all four serotypes circulated, the predominant serotype based on PCR and/or virus isolation $(n=194)$ was DEN-1 (150 [77\%] DEN-1, 36 [19\%] DEN-2, 4 [2\%] DEN-3 and 4 [2\%] DEN-4).

\section{The Lao People's Democratic Republic}

Under the National Surveillance System for Selected Notifiable Diseases (the Lao People's Democratic Republic Early Warning and Response Network), the 2009 dengue case classification system was incorporated in 2011 (Table 1), and all cases fulfilling the clinical criteria are reported. In 2011, the Lao People's Democratic Republic reported 3905 cases (seven fatal), with a peak in week 36 ( $n=188$ cases) in September (Figure 2). Among 111 laboratory-tested cases, 49 (44\%) were laboratory confirmed by ELISA. IgM-positive specimens for which the time between date of onset and date of collection is less than five days and IgG negative were selected for serotyping; among eight serotyped cases, the predominant serotype was DEN-1 (6 DEN-1, 1 DEN-2 and 1 DEN-3).

\section{Malaysia}

Under the National Notifiable Infectious Diseases system, all clinically suspected dengue cases, including dengue haemorrhagic fever and dengue shock syndrome, are reported (Table 1). In 2011, Malaysia reported 19884 cases (36 fatal). The highest number of cases $(n=596)$ was reported during week two in January; the weekly number of cases was overall low and stable throughout the year (Figure 2). Among 8105 laboratory-tested cases, 7301 (90\%) 
Figure 2. Reported number of dengue cases by calendar week (Cambodia, the Lao People's Democratic Republic, Malaysia and Singapore) or month (Australia and the Philippines), 2011
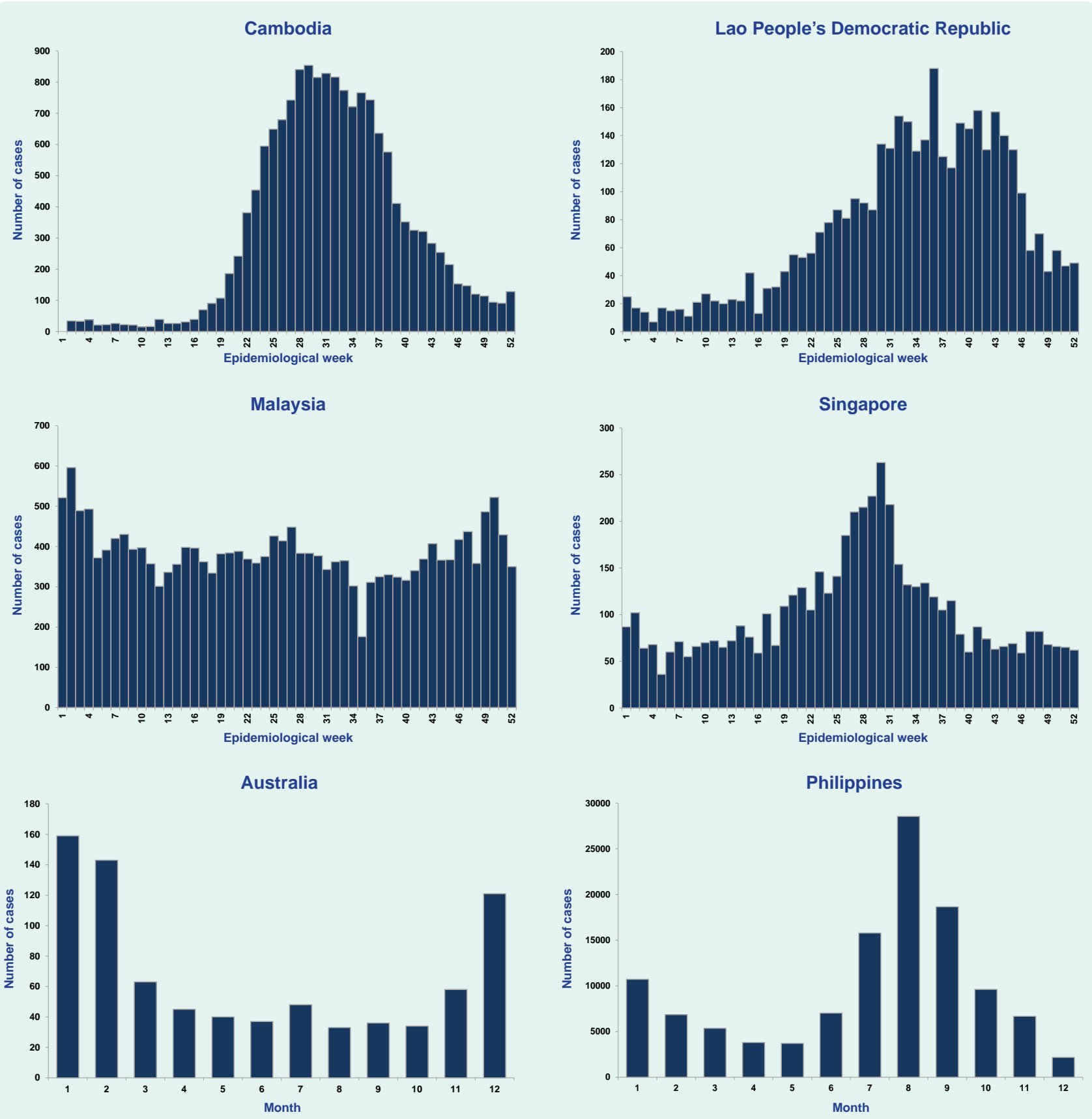

Source: World Health Organization Western Pacific Regional Office based on data provided by the Member States. Note: Units for the vertical axis is variable by country.

were confirmed by serology (IgG/lgM) or antigen detection (NS-1). Among the 235 serotyped cases, all four serotypes circulated (74 [32\%] DEN-1, 60 [25\%] DEN-3, 57 [24\%] DEN-2 and 44 [19\%] DEN-4).

\section{The Philippines}

Under the Philippines Integrated Disease Surveillance and Response, all clinically suspected and probable dengue cases are reported by the Department of Health's National Epidemiology Center (NEC) (Table 1). NEC is in the transition period for adopting new case definitions based on the 2009 dengue classification. In 2011, the Philippines reported 125975 cases (654 fatal) with a peak in the month of August ( $n=28$ 549) (Figure 2). Adolescent and adult males made up a higher proportion of the reported cases relative to females. Among 190 laboratory-tested cases, 190 (100\%) were 
confirmed by serology (IgM) and a limited number by PCR. Among the 88 serotyped cases, the predominant serotypes were DEN-1 and DEN-3 (39 [44\%] DEN-1, 38 [43\%] DEN-3 and 11 [13\%] DEN-2).

\section{Singapore}

Under the Infectious Diseases Management and Outbreak System, dengue cases are reported as dengue fever or dengue haemorrhagic fever cases. Laboratory testing occurs for all clinically suspected or probable cases, and only cases that are laboratory confirmed (by serology (IgM) or PCR/NS-1) are registered as dengue cases (Table 1). In 2011, Singapore reported 5330 cases (six fatal) with a peak in week 30 ( $n=263$ cases) in July (Figure 2). Adolescents and adult males made up a higher proportion of the reported cases relative to females. A proportion of the confirmed cases are serotyped; among the 712 serotyped cases, the predominant serotype was DEN-2 (549 [77\%] DEN-2; 75 [10\%] DEN-1, 61 [9\%] DEN-3 and 27 [4\%] DEN-4).

\section{Viet Nam}

Under the National Notifiable Disease Surveillance system, the 2009 dengue case classification system was incorporated in 2011, and all cases fulfilling the clinical criteria are reported (Table 1). In 2011, Viet Nam reported 69680 dengue cases (61 fatal). Laboratory testing occurs for a proportion of clinically suspected dengue cases ( $7 \%$ by serology and 3\% by virus isolation). Among 7249 laboratory-tested cases, 3262 (45\%) were confirmed by serology or virus isolation. Among the 674 serotyped cases, the predominant serotype was DEN-1 (284 [42\%] DEN-1, 217 [32\%] DEN-2, 118 [18\%] DEN-4 and 55 [8\%] DEN-3).

\section{Pacific subregion}

\section{Australia}

Under the National Notifiable Diseases Surveillance System, all clinically suspected dengue cases that are laboratory-confirmed are reported (Table 1). In 2011, Australia reported 820 cases (zero fatal; personal communication, Phil Wright, Office of Health Protection, Australian Commonwealth Department of Health and Ageing) with a peak in the month of January ( $n=158$ cases) (Figure 2). The predominant serotype detected in North Queensland, among
69 locally acquired dengue cases, was DEN-2 (47 [68\%] DEN-2, 13 [19\%] DEN-4 and 9 [13\%] DEN-1); 34 of 67 (51\%) with gender information were male. Among 27 imported cases in North Queensland, nine were $D E N-1$, eight were $D E N-3$, seven were DEN-2, one was DEN-4 and two were unspecified; 17 of $27(63 \%)$ were male (personal communication, Gregor Divine, Tropical Regional Services, Health Services and Clinical Innovation Division, Queensland Health).

\section{The Federated States of Micronesia and the Marshall Islands}

More than 2000 cases were reported from the Federated States of Micronesia and the Marshall Islands both of which weer detected through event-based surveillance (Table 1). In Yap, a state of the Federated States of Micronesia, an outbreak started in September 2011 with more than 1000 clinically suspected cases (two deaths) reported by the end of the 2011 calendar year; the predominant serotype was DEN-2. The outbreak in the Marshall Islands started in October 2011 with more than 1000 clinically suspected cases (zero deaths) reported by the end of the 2011 calendar year; the predominant serotype was DEN-4.

\section{DISCUSSION}

In 2011, dengue continued to show high levels in the Western Pacific Region. While overall occurrence was lower than that of the previous year in the Asia subregion, Cambodia reported a considerably higher number of cases and CFR relative to 2010. In Cambodia, the Lao People's Democratic Republic, the Philippines and Singapore, peaks in dengue activity followed historic seasonal trends, occurring shortly after the onset of the rainy season from July to September, similar to that observed in 2010; overall DEN-1 appeared to predominate. In the Pacific subregion, the Federated States of Micronesia and the Marshall Islands experienced unusually large dengue outbreaks associated with different serotypes of DEN-2 and DEN-4.

The dengue levels in the Region, which are variable by country, season, year and serotype, highlight the need for continuous surveillance and information-sharing. Rapid information-sharing at the local level for vector control and case-based response are essential to 
interrupt transmission. Routine and timely informationsharing at the regional level helps to improve the countries' awareness and understanding of the dengue situation in neighbouring countries or those with close trade/travel links (e.g. in the isolated Pacific island countries and areas, dengue activity has been associated with introductions from Asia). ${ }^{9}$ Thus, sharing of regional information can feed into better-informed assessments and responses by each country such as timely enhanced awareness activities. Since routine biweekly reporting of the regional dengue situation was initiated in late $2010,{ }^{10}$ information has also been regularly disseminated through wider public health surveillance networks, such as ProMED. ${ }^{11}$ Regional surveillance data showing continued high level dengue levels led to the launch the Association of Southeast Asian Nations Dengue Day in June 2011 aimed at improving advocacy and community participation. The WHO Regional Office for the Western Pacific has been working on sharing additional surveillance data such as serotype and gender information that can further improve assessment activities; monitoring these data may inform of important changes in dengue epidemiology (e.g. who/where to target, herd immunity/susceptibility). ${ }^{4,12}$ For instance, young adult males in several dengue-endemic countries in the Region continued to have a higher reported number of cases relative to their female counterparts.

These surveillance data have important limitations both in interpreting the actual burden of dengue (e.g. underreporting of mild cases) and trends over time (e.g. changes in disease awareness, reporting behaviour and surveillance systems). Australia and Singapore report laboratory-confirmed cases only, but dengue surveillance in Cambodia, the Lao People's Democratic Republic, Malaysia, the Philippines and Viet Nam is based on clinically suspected cases; therefore, the true number of incident dengue cases may be under- or over-reported. The proportion of true dengue cases among clinically suspected cases may also differ across seasons due to differential physician awareness and reporting behaviour (e.g. during low versus high dengue season).

There have been notable changes in the surveillance systems in recent years. The new dengue case classification scheme was incorporated by Cambodia, the Lao People's Democratic Republic and Viet Nam in 2011; the Philippines surveillance system has been transitioning from a sentinel to an all-case reporting system since 2008. As dengue surveillance across countries differs, any comparison between countries should be interpreted with caution. CFRs are affected not only by clinical management but also by health care utilization, the reporting behaviours of clinicians, case definitions, follow-up and verification procedures. Sampling schemes for laboratory confirmation differ across countries and may not be systematic, limiting the interpretability of the reported serotype distribution. Due to late/incomplete reporting, these data are provisional and some countries will have final figures that are different from those reported here.

While acknowledging these limitations, as dengue epidemiology continues to evolve in the Region, at times unpredictably, there will continue to be a need for region-wide sharing of dengue data on a routine, timely basis. A dengue vaccine may become a reality in the near future, and there is even more reason to have continuous, reliable and systematic dengue surveillance to assist in evaluation of the vaccine once it is launched. Enhancement or implementation of dengue surveillance can act as an entry point for countries where surveillance capacities are limited for endemic infectious diseases, and such activities are in line with WHO's Asia Pacific Strategy for Emerging Diseases framework to strengthen national capacities for surveillance and response. Even in countries where dengue is not currently endemic (e.g. Japan), the ever-increasing importation of cases signifies the importance of monitoring and sharing dengue information across the Region. ${ }^{13}$ While dengue continues to burden our Region, we hope that regional information-sharing can contribute to countering the threat.

\section{Conflicts of interest}

None declared.

Funding

None.

\section{Acknowledgements}

The authors would like to thank all the WHO Western Pacific Regional Country Office staff and Member States for assisting wiht the surveillance data collection and reporting. 


\section{References:}

1. Kyle JL, Harris E. Global spread and persistence of dengue. Annual Review of Microbiology, 2008, 62:71-92. doi:10.1146/annurev. micro.62.081307.163005 pmid:18429680

2. Cummings DA et al. Travelling waves in the occurrence of dengue haemorrhagic fever in Thailand. Nature, 2004, 427:344-347. doi:10.1038/nature02225 pmid:14737166

3. Ler et al. Epidemiological characteristics of the 2005 and 2007 dengue epidemics in Singapore - similarities and distinctions. Western Pacific Surveillance and Response, 2011, 2:e1. doi:10.5365/wpsar.2010.1.1.011

4. Anker M, Arima Y. Male-female differences in the number of reported incident dengue fever cases in six Asian countries. Western Pacific Surveillance and Response, 2011, 2:e1. doi:10.5365/wpsar.2011.2.1.002

5. Suaya JA et al. Cost of dengue cases in eight countries in the Americas and Asia: a prospective study. The American Journal of Tropical Medicine and Hygiene, 2009, 80:846-855. pmid:19407136

6. Huy R et al. Cost of dengue and other febrile illnesses to households in rural Cambodia: a prospective community-based case-control study. BMC Public Health, 2009, 9:155. doi:10.1186/14712458-9-155 pmid:19473500

7. Arima $Y$, Matsui $T$. Epidemiologic update on the dengue situation in the Western Pacific Region, 2010. Western Pacific
Surveillance and Response, 2011, 2:e1. doi:10.5365/ wpsar.2011.2.2.005

8. Hanna JN, Ritchie SA. An apparent recent decline in importations of dengue from Papua New Guinea into north Queensland. Communicable Diseases Intelligence Quarterly Report, 2009, 33:34-5. pmid:19618767

9. Wilder-Smith A, Gubler DJ. Geographic expansion of dengue: the impact of international travel. Medical Clinics of North America, 2008, 92:1377-1390, x. doi:10.1016/j.mcna.2008.07.002 pmid:19061757

10. Dengue Situation Updates. Manila, World Health Organization Regional Office for the Western Pacific, 2012 (http://www.wpro. who.int/emerging_diseases/DengueSituationUpdates/en/index. html, accessed 9 November 2012).

11. Dengue/DHF update 2012. ProMED, 2012 (http://www. promedmail.org/direct.php?id=20120917.1297396, accessed 9 November 2012).

12. Lee KS et al. Dengue virus surveillance for early warning, Singapore. Emerging Infectious Diseases, 2010, 16:847-849. doi:10.3201/eid1605.091006 pmid:20409381

13. Nakamura $\mathrm{N}$ et al. Incidence of dengue virus infection among Japanese travellers, 2006 to 2010. Western Pacific Surveillance and Response, 2012, 3:1. doi:10.5365/ wpsar.2011.2.3.002 